\title{
28 Research Square \\ Reference Intervals of Renal Function Set Established for Healthy Pregnant Women
}

\section{Mingyang Wu}

XiangYa School of Medicine of Central South University

Wenen Liu

Xiangya Hospital Central South University

Zhongyuan Xiang ( $\nabla$ xiangzhongyuan@csu.edu.cn )

Department of Laboratory Medicine, The Second Xiangya Hospital of Central South University

\section{Research Article}

Keywords: reference interval, renal function set, pregnancy complications, pregnant women, trimester of pregnancy, eclampsia

Posted Date: February 18th, 2022

DOI: https://doi.org/10.21203/rs.3.rs-1267597/v1

License: @ (i) This work is licensed under a Creative Commons Attribution 4.0 International License. Read Full License 


\section{Abstract \\ Objective}

This cross-sectional study aims to explore variation trend of renal function for healthy pregnant women at different gestational age, and to establish RIs of renal function set tests according to trimester of pregnancy.

\section{Methods}

A total of 120 healthy pregnant women and 40 healthy non-pregnant women were enrolled, divided into early trimester ( $1-13$ weeks of gestation, $n=40)$, second trimester ( $14-27$ weeks of gestation, $n=40)$, third trimester ( $\geq 28$ weeks of gestation, $n=40)$, and non-pregnant women group $(n=40)$. Analytes of $U A, B U N$, $\mathrm{Cr}, \beta 2-\mathrm{MG}$, Cys-C, RBP, NAG, CO2, HCY, CG, C1q and NGAL were measured using the analytical systems in Second Xiangya Hospital. The Rls were defined using non-parametric $95 \%$ intervals.

\section{Results}

The Rls for $\mathrm{UA}, \mathrm{Cr}, \beta 2-\mathrm{MG}, \mathrm{NAG}, \mathrm{CO} 2$, Hcy and $\mathrm{C} 1 \mathrm{q}$ were established respectively for the first and second trimester group and the third trimester group, there are huge differences after pregnancy. The RIs for BUN was different for 1-13 weeks and after 14 weeks. There are significant differences for NGAL for pregnant and non-pregnant women but not for Cys-C and RBP.

\section{Conclusion}

The Rls for renal function tests in pregnant women were established, thus providing clinical reference intervals for the clinicians.

\section{Introduce}

For the purpose of making a medical diagnosis, therapeutic management decision, or other physiological assessment, a measured or observed laboratory test result from a person (usually a patient) is compared with a reference interval.[1] Traditionally, reference intervals are acquired from supposedly healthy subjects. However, health is not a well-defined condition and has come to be regarded as relative. It can be difficult, however, to define the appropriate reference interval for any particular laboratory test. Many factors, including age, race, gender, posture during specimen collection, geographical location, diurnal variations, and even seasonal changes may influence the results of laboratory tests[2]. These factors are partially responsible for the intro-and-inter-individual variations observed in the results of laboratory tests, and reference intervals should reflect these variations[3]. 
However, physiological changes always occur during pregnancy, which can influence biochemical parameters $[4,5]$, here we would like to focus on how renal function tests change during pregnancy. Clinical interpretation for kidney function tests: UA is the metabolic end product of purine nucleotide, which is excreted from feces and urine. The concentration of UA is an important biochemical marker of renal dysfunction and has been associated with the development of hypertension[6]. Serum UA can be used as an indicator to judge the damage of PE (preeclampsia) renal function and is also a good predictor of fetal/neonatal outcomes in them[7].The increase of serum UA, $\mathrm{Cr}$ and BUN levels may indicate impaired renal function in patients with hypertension, which have certain application value in evaluating and monitoring the degree of impaired renal function in pregnancy[8]. Cys- $\mathrm{C}$ is a kind of cysteine protease inhibitors, its generation isn't affected by gender, age, muscle mass, diet, inflammation, bilirubin, hemolysis; it has no obvious circadian rhythm change, no renal tubular secretion. The kidney is the only organ in the scavenging cycle of Cys-C, and its serum concentration is mainly determined by renal function, which has its unique advantages in reflecting glomerular filtration function[9]. Serum Cys$C$ test for gestational hypertension disease has good diagnostic value of renal impairment, when combined with detection of serum Cys-C, BUN, and $\mathrm{Cr}$, which is advantageous to the HDCP (hypertensive disorder complicating pregnancy) and concurrent renal damage diagnosis and monitoring[6]. $\beta_{2}-\mathrm{MG}$ is a low molecular weight protein with a molecular weight of only 11800 , because the electrophoretic diagram is located in the band of the $\beta_{2}$, so it is called the $\beta_{2}$-microglobulin, which enters the blood circulation during metabolism, passes through the capillary wall of the glomerulus at liberty and is almost completely reabsorbed and decomposed by the renal tubules under normal conditions. It is only decomposed and excreted by the kidney and the day and night changes are very small. Therefore, it can be used as an indicator to assess the functional status of glomerulus and renal tubules[10]. $\beta 2$ microglobulin ( $\beta 2-\mathrm{m})$ is a major component of the amyloid in dialysis-related amyloidosis (DRA) .[11]The cystatin $C$ and $\beta_{2} \mathrm{M}$ are not only mainly increased in parallel in the third trimester of normal pregnancy, but even more so in diagnosing pre-eclampsia with similar diagnostic capacity. [12]NAG is an enzyme that is widely found in various tissues organs, body fluids and blood cells, and is an acidic hydrolytic enzyme in lysosomes. The determination of serum NAG activity is more sensitive to renal parenchymal lesions, especially acute and active lesions, and is mainly used for the monitoring and observation of early renal injury. [4]In patients with pregnancy induced hypertension, the serum levels of NAG, mAlb and Cys $C$ increases with the aggravation of the disease, and the combined detection is helpful to the early detection of pregnancy induced hypertension with renal impairment[13]. Under certain temperature and pressure, the determination of the amount of carbon dioxide in dissolving to the plasma or serum is $\mathrm{CO}_{2}$, which is mainly used to estimate the content of sodium bicarbonate in blood, to assess the imbalance of acid-base balance and its degree, and to determine the function of regulating the balance of acid and alkali in the kidney[14]. Hcy is an amino acid containing sulfur, which is demethylated by methionine and is an intermediate product of methionine metabolism and excretes from the kidneys[15]. The characteristics of blood, metabolic system and circulating metabolism of Hcy in pregnancy make plasma Hcy level closely related to the occurrence and development of pregnancy-related diseases[16]. Complement $\mathrm{C} 1 \mathrm{q}$ is an important component of complement C1.[17] Studies have shown that the absence of serum complement $\mathrm{C} 1 \mathrm{q}$ (including lack of component and functional disorder) will cause the 
body's immune complex deposition in tissue, causing immune complex diseases, such as SLE, glomerulonephritis[18], etc. It is found that NGAL is closely related to the occurrence of chronic kidney disease and can be an important marker for early renal injury $[13,19]$. NGAL, as a new member of lipocalin family, is a fat cytokine and an inflammatory factor. NGAL participates in different physiological and pathological process in the body, such as embryonic development, cell differentiation, apoptosis, tumor occurrence and development, inflammatory immune response, glycolipid metabolism and insulin resistance. The biological function of NGAL plays an important role in pregnancy related diseases such as hypertensive disease, GDM and preterm birth[20]. (Full names and abbreviations of common renal function are uric acid (UA), blood urea nitrogen (BUN), serum creatinine ( $(\mathrm{Cr} r), \beta_{2}$-microglobulin $\left(\beta_{2}-\mathrm{MG}\right)$, cystatin-C (Cys-C), retinol binding protein (RBP), N-acetyl- $\beta$-D-glucosidase (NAG), homocysteine (HCY), Complement1q (C1q), neutrophil gelatin-related apolipoprotein ( $\mathrm{NGAL})$, carbon dioxide $\left(\mathrm{CO}_{2}\right)$. An understanding of these changes is important for the management of the health of pregnant women, as pathological deviations are not able to be distinguished from normal physiological alterations. Thus, it is important that the testing conditions used to collect the reference data be closely matched with the testing conditions used for patient data[21].

Therefore, it's necessary to establish appropriate reference intervals for renal function set for the first, the second and the third trimester of pregnancy, for the purpose of making a medical diagnosis, therapeutic management decision, or other physiological assessment which belong to pregnancy women.

\section{Materials And Methods}

\subsection{Source of reference population}

Ethical Considerations This study was approved by the Ethics Committee of the Second Xiangya Hospital of Central South University, and all the subjects gave informed written consent. All experiments were performed in accordance with relevant guidelines and regulations.

Source of population A multi-level cluster sampling approach was used to recruit healthy individuals (factors such as age, gravidity, gestational age were taken into account) from Changsha. For this study, the target population was all Han Chinese women.

Exclusion criteria To select healthy individuals, the following exclusion criteria based on the CLSI C28-A3 document ${ }^{[1]}$ was used:

1. A diagnosis of chronic disease, hepatic disease, or endocrine disease;

2. A history of inherited diseases;

3. Body mass index (BMI) $\geq 28 \mathrm{~kg} / \mathrm{m}^{2}$ or $\leq 18.5 \mathrm{~kg} / \mathrm{m}^{2}$;

4. High blood pressure for longer than 3 years (either systolic pressure $\geq 140 \mathrm{~mm} \mathrm{Hg}$ or diastolic pressure $\geq 90 \mathrm{mmHg}$ );

5. Any abnormality of the heart, liver, lungs, and kidney structure as found using ultrasonic wave or abnormal electrocardiogram; 
6. Surgery during the previous 4 months of pregnancy or blood transfusion or donation within 6 months;

7. Drug intake within 2 weeks or antibiotic abuse;

8. Excessive smoking ( $>20$ cigarettes per day) or drinking ( $>30 \mathrm{~g}$ per day);

9. Heavy exercise or laborious work;

10. The following exclusionary laboratory test results: triglyceride $\geq 2.26 \mathrm{mmol} / \mathrm{L}$, total cholesterol $\geq 6.22$ $\mathrm{mmol} / \mathrm{L}$, fasting blood glucose $>7.0 \mathrm{mmol} / \mathrm{L}$, hepatitis $B$ surface antigen, anti-hepatitis $C$ virus or anti-HIV positive, abnormal urinalysis, red cell count $\geq 5.0 \times 10^{12} / \mathrm{L}$ or $\leq 3.0 \times 10^{12} / \mathrm{L}$, hemoglobin $\leq 110 \mathrm{~g} / \mathrm{L}$, white cell count $\geq 12.0 \times 10^{9} / \mathrm{L}$ or $\leq 4.0 \times 10^{9} / \mathrm{L}$, platelet count $<100 \times 10^{9} / \mathrm{L}$.

With age ranged from 20 to 37 years old, 120 pregnant women and 40 non-pregnant women met the exclusion criteria finally.

\subsection{Laboratory analysis}

Sample collection and handling. The blood samples were collected from the cubital vein and dispensed into $5 \mathrm{ml}$ in ethylene diaminetetra-acetic acid vacutainer tubes. All blood samples were centrifuged to separate serum at $3500 \mathrm{~g}$ for 5 minutes before analyzing, and all analyses were performed on fresh samples. All analyses were performed within 2 hours after collection, and finally preserved at 4 centigrade refrigerator. The collection and handling of specimens was executed procurement at room temperature (25 degree). All blood samples were come from the clinical laboratory in the Second Xiangya Hospital of Central South University.

Assay of renal function tests. The clinical laboratories in the Second Xiangya Hospital were qualified by the National External Quality Assessment of China. Concentrations of renal function tests set were assayed with the HITACHI 7600-120 analyzer (HITACHI, Japan) according to the manufacturer's instructions. Before sample analysis stage, quality control of measurement equipment was performed and proved to be qualified.

Processing of test results. Results obtained by measurement of reference individuals were directly used for establishment of reference intervals, if the relative bias between the means of measured values of reference materials obtained by the analytical systems and the assigned target values of reference materials meet the desirable specification for inaccuracy of biologic variation [17].

\subsection{Statistical methods}

SPSS V.20.0 software (SPSS, Inc., Chicago, Illinois, USA) and Graphpad Prism V.5.0 software were used for data analysis. The statistical methods recommended in the CLSI C28-A3 document were used to define RIs ${ }^{[18]}$. The RIs were defined by non-parametric $95 \%$ intervals, and the lower limit and upper limit of Rls were respectively, expressed as the 2.5th and 97.5 th percentiles of the measurands levels; the $90 \%$ confidence intervals $(90 \% \mathrm{Cl})$ of the lower and upper reference limits were calculated by non-parametric method. According to CLSI C28-A3 [1], the Dixon test method was used to eliminate outliers. Non-normally distributed data were normalized, and normal transformation was performed using reciprocal 
transformation and square root transformation. The independent sample $T$ test was applied to compare difference between two groups; differences with $p<0.01$ were regarded to be statistically significant. ANOVA analysis with Tukey's test was adopted for pair-wise comparison, to compare the serum renal function tests levels among three groups; $p<0.05$ was considered to be significantly different, suggesting that the RIs of pregnant women should be established apart according to gestational age. For those two groups that $p>0.05$, we merged.

\section{Results}

\subsection{Reference population}

The baseline characteristics of the inclusive women in this study are shown in Table 1.

Table 1

Characteristics of the reference population enrolled in this study.

\begin{tabular}{|llll|}
\hline Qualitative Variable & Total(N) & Non-pregnant $(\mathbf{N})$ & Pregnant $(\mathbf{N})$ \\
\hline Total & 160 & 40 & 120 \\
\hline Age group/years old & & & \\
\hline $20-25$ & 50 & 14 & 36 \\
\hline $26-31$ & 69 & 13 & 56 \\
\hline $32-37$ & 41 & 13 & 28 \\
\hline Quantitative Variable & Mean \pm SD & Mean $\pm S D$ & Mean $\pm S D$ \\
\hline Age/years old & $30.1 \pm 8.4$ & $32.6 \pm 9.2$ & $28.8 \pm 6.3$ \\
\hline
\end{tabular}

\subsection{Reference intervals for renal function tests sets of non-pregnant women and pregnancy women in different gestational ages}

Tables 2 summarized the reference intervals for the renal function tests of non-pregnancy women and pregnancy women in different gestational ages.

\subsection{The changes of serum renal function tests levels between non-pregnancy women and pregnancy women in different gestational ages}

The results of the Kolmogorov-Smirnov test showed that the $\mathrm{UA}, \mathrm{BUN}, \mathrm{CR}, \mathrm{CO}_{2}$ and $\mathrm{C} 1 \mathrm{q}$ levels of healthy pregnant women and non-pregnant women were normally distributed ( $p>0.05)$, but the $\beta_{2}-M G, C Y S-C, R B P$, NAG, HCY and NGAL level. The distributions of biochemistry parameters above of the non-pregnant and pregnant women groups became normal after transformation. 
The outcomes of independent sample T test suggested that UA $(t=5.99, p<0.01), B U N(t=-9.70, p<$ $0.01), C R(t=-3.98, p<0.01), \beta_{2}-M G(t=-2.73, p<0.01), N A G(t=9.31, p<0.01), \mathrm{CO}_{2}(t=-7.77, p<0.01)$, Hcy $(t=-7.84, p<0.01), C 1 q(t=-21.95, p<0.01), N G A L(t=5.75, p<0.01)$ confirmed significant differences between non-pregnant and pregnant groups, which suggesting that RIs for UA, BUN, CR, $\beta 2$ $\mathrm{MG}, \mathrm{NAG}, \mathrm{CO}_{2}, \mathrm{Hcy}, \mathrm{C} 1 \mathrm{q}$ and $\mathrm{NGAL}$ in non-pregnant and pregnant healthy women should be respectively established. However, no significant differences were observed in CYS-C $(t=-5.66, p>0.01)$ and RBP $(t=$ $0.46, p>0.01)$.

We also observed a significant increasing trend in UA, NAG and NGAL reference intervals and a significant decreasing trend in $\mathrm{BUN}, \mathrm{Cr}, \beta_{2}-\mathrm{MG}, \mathrm{CO}_{2}, \mathrm{Hcy}$ and $\mathrm{C} 1 \mathrm{q}$ reference intervals from the first trimester to the third trimester (Fig. 1).

\subsection{Comparison of reference intervals among pregnant healthy women in different gestational ages}

The results of the Kolmogorov-Smirnov test showed that serum UA, BUN, $\mathrm{Cr}, \mathrm{Cys}-\mathrm{C}, \mathrm{RBP}, \mathrm{CO}_{2}$ and $\mathrm{C} 1 \mathrm{q}$ levels in the first, second, and third trimesters groups were distributed normally ( $p>0.05$ ), but the $\beta 2-M G$, NAG, Hcy, CG and NGAL levels during the first, second, and third trimesters were not distributed normally $(p<0.05)$; the $\beta 2-M G, N A G, H c y, C G$ and NGAL values of the three groups were normally distributed after normal transformation. The Levene test indicated equal variance. ANOVA confirmed that differences for the following serum renal function parameters of healthy women in the three groups showed statistically significant: UA ( $F=60.108, p<0.05), B U N(F=11.385, p<0.05), \operatorname{Cr}(F=3.906, p<0.05), \beta 2-M G(F=$ 27.315, $p<0.05), N A G(F=119.249, p<0.05), \mathrm{CO}_{2}(F=12.590, p<0.05)$, Hcy $(F=5.165, p<0.05), C 1 q(F$ $=14.023, p<0.05)$; but the differences for NGAL among three groups showed no significant $(F=0.021, p$ $>0.05$ ). Tukey's test was adopted for pairwise comparison: the serum UA levels of the first and second trimester groups did not significantly differ $(p=0.982)$, whereas those in the first and third trimester groups and those in the second and third trimester groups did significantly differ (both $p<0.05$ ). The results of the first and second trimester groups were combined into one group. The RIs for UA in pregnant healthy women according to trimester are shown in Table 3. Same as above mentioned, the significances of other parameters are summarized in Table 4.

Table 2 reference intervals for renal function tests of pregnancy women in different gestational ages (the 2.5th and 97.5th values) and non-pregnancy women. 


\begin{tabular}{|c|c|c|c|c|c|c|c|c|c|c|c|c|}
\hline & Group & ua & Bun & $C R$ & $\beta 2+M G$ & CYsC & RBP & NAG & $\cos$ & $\mathrm{HCY}$ & $\mathrm{Clq}$ & NGAL \\
\hline \multirow{3}{*}{ Reference Interval } & $<14$ weeks & $149.40-289.90$ & 1.734 .25 & $33.16-61.27$ & $0.90-2.08$ & $0.43-0.69$ & $30.82 \cdot 49.37$ & $19.02-41.90$ & $14.81-23.17$ & $5.96-15.88$ & $190.05-283.57$ & 124.63 .559 .84 \\
\hline & $x=28$ weels & $211.05-374.43$ & 2.384 .84 & $33.57-68.95$ & $1.17-2.50$ & $0.46-1.25$ & 28.43 .56 .50 & $34.31-83.61$ & $12.52-24.09$ & $6.12-16.75$ & $166.00-258.38$ & $147.67-635.12$ \\
\hline & non-pregnancy & $180.42-366.81$ & 2.92 .7 .22 & $40.11-69.14$ & $1.03-2.83$ & $0.5-0.99$ & 22.44 .50 .99 & $12.11 \cdot 24.28$ & $16.82-25.19$ & $8.25-22.92$ & $280.27 \cdot 389.20$ & $51.52-387.08$ \\
\hline \multirow{4}{*}{25 th $(90 \%(1)$} & s14 weeks & $149.40(149.40-150.51)$ & $1.73(1.73-2.02)$ & $33.16(33.10-37.31)$ & $0.90 \mid 0.9-0.97)$ & $0.43(0.43-0.45)$ & $30.82(30.80-32.00)$ & $19.02(19.00-22.27)$ & $14.81(14.80-15.70)$ & $5.96(5.95-6.76)$ & $190.05(189.80-200.33)$ & $124.63(124.60-149.10)$ \\
\hline & $15-27$ weeks & $126.30(126.30-148.74)$ & $1.97(1.96-2.27)$ & $35.58(35.50-40.70)$ & $0.79(0.79-0.96)$ & $0.40(0.41-0.46)$ & $28.21(28.20-28.90)$ & $24.72(24.70-26.26)$ & $14.33(14.30-15.81)$ & $5.32(5.31-6.51)$ & 181.74 (181. $50-195.27)$ & $139.25(139.20-145.19)$ \\
\hline & $x=28$ weels & $211.05(211.00-228.54)$ & $2.38(2.38-2.80$ & $33.57(33.50-39.02)$ & $1.17(1.17-1.93)$ & $0.45(0.46-0.69)$ & $28.43(28.40-30.91)$ & $34.31(34.30-39.96)$ & $12.52(12.50-13.50)$ & $6.12(5.12-6.66)$ & $166.00(165.90-174.55)$ & $147.67(147.50-161.75)$ \\
\hline & non-pregnancy & $180.42(179.8-192.6)$ & $2.92(2.92-3.20)$ & $40.11(40.141 .40)$ & $1.03(1.03-1.20)$ & $0.5(0.50-0.57)$ & $22.44(22.40-25.94)$ & $12.11(12.10-13.01)$ & $16.82(16.80-17.71)$ & $8.25(8.24-9.06)$ & $280.27(280.20-283.72)$ & $51.52(51.13-89.05)$ \\
\hline \multirow{4}{*}{ 97.5th(90\%6) } & <14 weeks & $289.90(250.24-290.42)$ & $4.25(3.654 .26)$ & $61.27(51.98-51.4)$ & $2.08(1.59-2.08)$ & $0.69(0.54 .0 .70)$ & $49.37(45.80-49.40)$ & $41.90(40.94-41.90)$ & $23.17(23.04 \cdot 23.20)$ & $15.88(10.27-15.90)$ & $283.57(265.94 \cdot 283.60)$ & $559.84(482.39-560.40)$ \\
\hline & 15-27 weeks & $281.40(271.90-281.41)$ & $4.90(4564.91)$ & $58.87(54.38 .58 .90)$ & $1.96(1.91-1.97)$ & $0.77(0.71-0.77)$ & $38.89(38.01-38.90)$ & $47.21(43.28 .47 .30)$ & $24.17(21.81-24.20)$ & $12.27(11.85-12.28)$ & $273.32(263.48 \cdot 273.50)$ & $574.77(453.28 .575 .90)$ \\
\hline & $x=28$ weels & $374.43(367.29-374.50)$ & $4.84(4.684 .84)$ & $68.95(59.78 .69 .10)$ & $2.50(2.18 \cdot 2.50)$ & $1.25(1.22-1.25)$ & $56.4(47.45-56.70)$ & $83.61(80.04-83.7)$ & $24.09(18.89-24.20)$ & $16.75(13.54-16.77)$ & () $258.38(240.18 \cdot 258.60)$ & $635.12(440.94-639.20)$ \\
\hline & non-pregnancy & $366.81(346.9-367.7)$ & $7.22(6.63-7.23)$ & $69.14(52.74-59.2)$ & $2.83(2.78-2.83)$ & $0.99(0.96-9.99)$ & $50.99(43.94-50.99)$ & $24.28(22.87-24.30)$ & $25.19(24.28-25.20)$ & $22.92(20.71-22.95)$ & $389.20(379.23 \cdot 389.45)$ & $387.08 / 327.90-387.90)$ \\
\hline
\end{tabular}

$U A$, uric acid (umol/l); BUN, urea nitrogen (mmol/l); $C r$, creatinine (umol/l); $\beta 2-M G, \beta 2$-microglobulin (mg/l); Cys-C, cystatin-C (mg/l); RBP, retinol binding protein ( $m g / l) ; N A G, N$-acetyl- $\beta$-D-glucosidase (u/l); HCY, homocysteine (umol/I); C1q,complement C1q (ng/l); NGAL, neutrophil gelatin-related apolipoprotein ( $\mathrm{ng} / \mathrm{l})$;CO2, carbon dioxide (mmol/l).

Table 3 P Values in Tukey's test for renal function of pregnant healthy women according to trimester

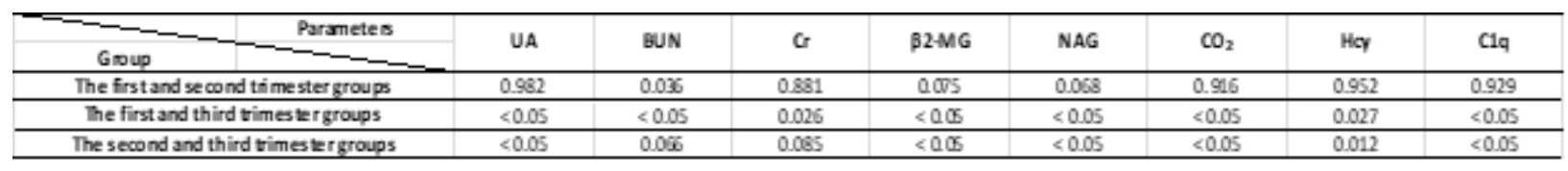

Table 4 Rls for renal function tests of pregnant healthy women according to trimester

\begin{tabular}{|c|c|c|c|c|}
\hline Parameter & Group & 2.5th and $90 \% \mathrm{Cl}$ & 97.5th and $90 \% 6 \mathrm{Cl}$ & Reference interval \\
\hline \multirow{2}{*}{ UA } & The first and second trimester groups(1-27we eks) & $131.188(130.300-149.571)$ & $290.333(278.812-298.400)$ & $131.188-290.333$ \\
\hline & The third trimestergroups (>-28weeks) & $211.050(211000-228.539)$ & $374.428(367.287-374.500)$ & $211.050-374.428$ \\
\hline \multirow{2}{*}{ BUN } & The first trimester groups(1-13weeks) & $1.732(1.730-2.016)$ & $4.248(3.647-4.260)$ & $1.732-4.248$ \\
\hline & The second and third trimester groups(14-28we eks) & $2.191(1.960-2.286)$ & $4.778(4.604-4.840)$ & $2.191-4.778$ \\
\hline \multirow{2}{*}{$\mathrm{Cr}$} & The first and second trimester groups(1-27weeks) & $35.503(33.100-37.802)$ & $58.865(55.295-61.400)$ & $35.508-58.865$ \\
\hline & The third trimestergroups (>-28weeks ) & $33.570(33.500-39.02 .1)$ & $68.945(59.783-69.100)$ & $33.570-68.945$ \\
\hline \multirow{2}{*}{$\beta 2-M G$} & The first and second trimestergroups (1-27weeks) & $0.900(0.790-0.958)$ & $1.970(1.910-2.080)$ & $0.900-1.970$ \\
\hline & The third trimester groups (>-28weeks) & $1.170(1.170-1.210)$ & $2.497(2.170-2.500)$ & $1.170-2.497$ \\
\hline \multirow{2}{*}{ NAG } & The first and second trime ster groups(1-27weeks) & $19.655(19.000-22.300)$ & $43.690(41.683-47.295)$ & $19.655-43.690$ \\
\hline & The third trimester groups (>-28weeks) & $34.308(34.300-35.364)$ & $83.613(74.469-83.700)$ & $34.308-83.613$ \\
\hline \multirow{2}{*}{$\mathrm{cos}$} & The first and second trimester groups(1-27we eks) & $14.808(14.300-15.795)$ & $23.193(21.800-24.199)$ & $14.808-23.193$ \\
\hline & The third trimester groups (>-28weeks) & $12.523(12.500-13.618)$ & $24.093(19.000-24.200)$ & $12.523-24.093$ \\
\hline \multirow{2}{*}{ Hcy } & The first and second trimester groups(1-27weeks) & $5.792(5.310-6.250)$ & $15.088(11.730-15.900)$ & $5.792-15.088$ \\
\hline & The third trimestergroups (>-28weeks ) & $6.125(6.120-6.660)$ & $16.745(14.504-16.770)$ & $6.125-16.745$ \\
\hline \multirow{2}{*}{$\mathrm{Clq}$} & The first and second trimester groups(1-27we eks) & $187.363(181.600-195.568)$ & $282.153(272.258-283.598)$ & $187.363-282.153$ \\
\hline & The third trimester groups ( $>-28$ weeks $)$ & $165.993(165.900-174.395)$ & $258.375(246.224-258.600)$ & $165.993-283.375$ \\
\hline
\end{tabular}

$U A$, uric acid (umol/l); BUN, urea nitrogen (mmol/l); $C r$, creatinine (umol/l); $\beta 2-M G, \beta 2$-microglobulin (mg/l); NAG, N-acetyl- $\beta$-D-glucosidase (u/l); CO2,carbon dioxide (mmol/l); HCY, homocysteine (umol/l); C1q,complement C1q (ng/l). 


\section{Discussion}

Overall, we found a significant increase in UA, NAG and NGAL reference intervals and a significant decrease in $\mathrm{BUN}, \mathrm{Cr}, \beta_{2}-\mathrm{MG}, \mathrm{CO}_{2}$, Hcy and $\mathrm{C} 1 \mathrm{q}$ reference intervals from the first trimester to the third trimester, and established RIs for pregnant women according to trimester and non-pregnant women, respectively.

Although there are important discoveries revealed, there are also limitations. First, standard design of surveys of large sample Rls of serum levels of renal function indexes for pregnant women is an ideal method to establish the reference but also a heavy project and infinitive within the remit of laboratories. Second, according to the recently updated CLSI / IFCC, it is considered impossible and unnecessary for most laboratories to establish their own reference intervals, and it is recommended to use the reference intervals established by conversion and verification. Third, the selection criteria of the population and the precision of the detection system is different, so the established reference intervals have certain limitations. However, compared with common Rls applied to all women, the established RIs for kidney function for pregnant women according to trimester is more realistic in the diagnosis of pregnancy complications.

Collectively, our results appear consistent with most reports[2, 3, 5, 6, 8, 20,22] associated with changes in serum levels of renal function of pregnant women.In pregnancy, oncotic pressure is substantially decreased as a result of expansion of the plasma volume, thus contributing to a rise in GFR.During pregnancy, the GFR increases $50 \%$ compared withnonpregnancy levels.[23] During the first period of pregnancy, the blood serum of UA in pregnant women decreased slightly, and then gradually increased to the per-pregnancy level. The reason is that the removal rate of UA in pregnant women is doubled, the increase of extracellular fluid, especially circulating blood volume, is effective in preventing the reabsorption of urinary acid in renal tubules. or a combination of both .Values equal to or greater than 6 $\mathrm{mg} / \mathrm{dL}$ of serum uric acid in patients with severe preeclampsia may be a valuable biomarker for preeclampsia and an association with the predict of adverse fetal and maternal effects.[24] Serum NAG in pregnant women was related to the formation and maturation of placenta. In the 1960s, when pregnancy was reported, NAG mainly existed in the decidual and chorionic cells of the placenta. Serum NAG began to increase after four months of gestation, especially in isoenzyme A form [25] which was parallel to the development of the placenta and the histological changes of the placental connective tissue after 4 months of gestation[26]. NAG was released to the maternal circulation mainly from the decidua and only partly from the amnion. Lysosomal enzyme release occurs prior to the onset of labor, it may play a role primarily in the ripening of the cervix [27]Previous studies have shown that NGAL participated in a variety of biochemical processes involving embryonic development and cell differentiation, this may be the reason why there were significant differences between pregnant women and non-pregnant women. there was no variation of serum NGAL values through the whole pregnancy and is consistent with the study by D'Anna $R,[20]$ During the late pregnancy, the sensitivity of osmoceptor to antidiuretic hormone dropped This change may be mediated by increased $\beta$-human chorionic gonadotropin, a pattern also seen to a lesser degree in menstruating women during the luteal 
cycle[28]and then led to retention of sodium and water and further caused GFR rose, which increased with the raised blood volume and renal blood flow (RBF); so the excretion of urea nitrogen and creatinine would increase and then the level of urea nitrogen and creatinine in serum would decrease, it's accordance with findings in this report that a significant decrease in BUN, Cr. Reference intervals of serum urea nitrogen and creatinine for healthy pregnant women, therefore, are lower than the that of health nonpregnant women, the general RIs of serum urea nitrogen and creatinine are no longer suitable for healthy pregnant women, it can be confirmed from the above results. The concentration of $\mathrm{C} 1 \mathrm{q}$ in serum of pregnant women decreased significantly. This may be due to anti-complement effect of normal pregnant women to make $\mathrm{C} 1 \mathrm{q}$ antibody in serum increased, $\mathrm{C} 1 \mathrm{q}$ combined with the $\mathrm{C} 1 \mathrm{q}$ antibody gradually led to the consumption of C1q serum and then levels of C1q dropped[29]. C1q localizes to the EVT and promotes its invasion, spiral artery remodeling and normal placentation at the feto-maternal interface [30] C1q produced by decidual endothelial cells (DECs) suggests that it acts as a link between the endovascular trophoblasts and spiral artery endothelial cells, involving the MAP kinase pathway via $\mathrm{gC1qR}$, a receptor for the globular head region of $\mathrm{C} 1 \mathrm{q}[31,32]$ Contrary to the findings of Zhang Yanling that Cys - $C$ and $\beta_{2}-M G$ higher in pregnant women than non-pregnant women, and the differences weren't statistically significant; in this study, a significantly decrease was found in $\beta_{2}-M G$, but no significant difference was observed in CYS-C. CYS-C secreted in the kidney, can be used as the indicator of the lesions in the permeability of glomerulus filtration [33] In Early-pregnancy Maternal serum cystatin $\mathrm{C}$ is an independent predictors of preeclampsia.[34]ß2-MG, serves as a sensitive indicator of the glomerular filtration rate and the reabsorption function of the proximal tubule that reflects the renal injury [13] However, serum Cys-C and $\beta_{2}-M G$ during pregnancy should decrease with the increase of glomerular filtration rate, to which $\beta_{2}-M G$ corresponded in this study. Different methods of measurement, sample sizes, and reference populations might also explain this. A significant decrease in Cys- $\mathrm{C}$ was found in this study, this is consistent with what has been found in previous report. Plasma Hcy levels began to decline in the early stages of pregnancy, and decreased to $50 \%-60 \%$ of the pre-pregnancy at $15-17$ weeks, and then stayed at this level until delivery, and returned to pre-pregnancy level after delivery[35]. lower homocysteine may be the protection of women from pregnancy complications and VTE, and thus lower homocysteine may contribute to maintaining homeostasis in haemostasis.[35]

There is room for improvement, for techniques as ultrasound and mammography are not available to pregnant women, some diseases cannot be ruled out. Therefore, reference individuals might not fully represent healthy pregnant women in our study. The population of this study was conducted by women in Changsha, and RIs need to be validated for other ethnic groups in the following studies.

In summary, RIs were established for UA, NAG and NGAL, BUN, $\mathrm{Cr}, \beta_{2}-\mathrm{MG}, \mathrm{CO}_{2}$, Hcy and $\mathrm{C} 1 \mathrm{q}$ in pregnant women according to the CLSI C28-A3 document. This study provides a valuable and reliable clinical reference for physicians.

\section{Declarations}




\section{Author Contributions}

M.W. and Z.X.: designed the research; Z.X.: conducted the research; W.L., Z.X.: collected the data; M.W.: analyzed the data and performed the statistical analysis; M.W.: wrote the manuscript; Z.X. edited the manuscript and had primary responsibility for final content; and all authors: read and approved the final version of the manuscript.

\section{Conflict of Interest}

The authors declare that the research was conducted in the absence of any commercial or financial relationships that could be construed as a potential conflict of interest.

\section{Notes}

The views expressed in this article are the personal views of the author(s) and may not be understood or quoted as being made on behalf of or reflecting the position of the regulatory agency/agencies or organisations with which the author(s) is/are employed/affiliated.

\section{Availability of Data and Materials}

The datasets used and analysed during the current study are available from the corresponding author on reasonable request.

\section{References}

1. Zhang, G.M., et al., Establishing Reference Intervals of Aspartate Aminotransferase-to-Platelet Ratio Index for Apparently Healthy Elderly. Clin Lab, 2016. 62(1-2): p. 135-40.

2. Fraser, C.G., Inherent biological variation and reference values. Clin Chem Lab Med, 2004. 42(7): p. 758-64.

3. Jones, G.R., Validating common reference intervals in routine laboratories. Clin Chim Acta, 2014. 432 : p. 119-21.

4. Hayashi, M., et al., Changes in urinary excretion of six biochemical parameters in normotensive pregnancy and preeclampsia. Am J Kidney Dis, 2002. 39(2): p. 392-400.

5. Burton, G.J., et al., Pre-eclampsia: pathophysiology and clinical implications. Bmj, 2019. 366: p. 12381.

6. Feig, D.I., Serum uric acid and the risk of hypertension and chronic kidney disease. Curr Opin Rheumatol, 2014. 26(2): p. 176-85.

7. Le, T.M., et al., Maternal serum uric acid concentration and pregnancy outcomes in women with preeclampsia/eclampsia. Int J Gynaecol Obstet, 2019. 144(1): p. 21-26.

8. Moghaddas Sani, H., S. Zununi Vahed, and M. Ardalan, Preeclampsia: A close look at renal dysfunction. Biomed Pharmacother, 2019. 109: p. 408-416. 
9. Tanaka, A., K. Suemaru, and H. Araki, A new approach for evaluating renal function and its practical application. J Pharmacol Sci, 2007. 105(1): p. 1-5.

10. Nishimoto, S., et al., A novel homocysteine-responsive gene, smap8, modulates mitogenesis in rat vascular smooth muscle cells. Eur J Biochem, 2003. 270(11): p. 2521-31.

11. Kambe, Y., et al., Artificial switching of the metabolic processing pathway of an etiologic factor, $\beta 2$ microglobulin, by a "navigator" molecule. Journal of controlled release : official journal of the Controlled Release Society, 2020. 327: p. 8-18.

12. Kristensen, $\mathrm{K}$., et al., Cystatin C, beta-2-microglobulin and beta-trace protein in pre-eclampsia. Acta Obstet Gynecol Scand, 2007. 86(8): p. 921-6.

13. Zhang, L., et al., The significance of combined detection of $C y s C$, urinary $m A / b$ and $\beta(2)-M G$ in diagnosis of the early renal injury in pregnancy-induced hypertension syndrome. Saudi J Biol Sci, 2019. 26(8): p. 1982-1985.

14. Gómez-Conde, S., et al., Molecular aspects and long-term outcome of patients with primary distal renal tubular acidosis. Pediatric nephrology (Berlin, Germany), 2021.

15. Gaiday, A.N., et al., Effect of homocysteine on pregnancy: A systematic review. Chem Biol Interact, 2018. 293: p. 70-76.

16. Wang, J., et al., Elevated circulating homocyst(e)ine levels in placental vascular disease and associated pre-eclampsia. Bjog, 2000. 107(7): p. 935-8.

17. Zwarthoff, S., et al., C1q binding to surface-bound IgG is stabilized by C1rs proteases. Proceedings of the National Academy of Sciences of the United States of America, 2021. 118(26).

18. Markowitz, G.S., et al., C1q nephropathy: a variant of focal segmental glomerulosclerosis. Kidney Int, 2003. 64(4): p. 1232-40.

19. Artunc-Ulkumen, B., et al., Relationship of neutrophil gelatinase-associated lipocalin (NGAL) and procalcitonin levels with the presence and severity of the preeclampsia. J Matern Fetal Neonatal Med, 2015. 28(16): p. 1895-900.

20. D'Anna, R., et al., Neutrophil gelatinase-associated lipocalin serum evaluation through normal pregnancy and in pregnancies complicated by preeclampsia. Acta Obstet Gynecol Scand, 2010. 89(2): p. 275-8.

21. Klee, G.G., Clinical interpretation of reference intervals and reference limits. A plea for assay harmonization. Clin Chem Lab Med, 2004. 42(7): p. 752-7.

22. Gómez-Conde, S., et al., Molecular aspects and long-term outcome of patients with primary distal renal tubular acidosis. Pediatr Nephrol, 2021.

23. Cheung, K.L. and R.A. Lafayette, Renal physiology of pregnancy. Adv Chronic Kidney Dis, 2013. 20(3): p. 209-14.

24. Moreno Santillan, A.A., J.C. Briones Garduño, and M.A. Diaz de Leon Ponce, Uric Acid in Pregnancy: New Concepts. Contrib Nephrol, 2018. 192: p. 110-115. 
25. Capodicasa, E., A. Angelini, and C. Tassi, Isoenzyme $A$ and urinary N-acetyl- $\beta$-D-glucosaminidase activity in normal pregnancy. Ren Fail, 2011. 33(6): p. 650-3.

26. Zou, S., et al., Use of data-independent acquisition mass spectrometry for comparative proteomics analyses of sera from pregnant women with intrahepatic cholestasis of pregnancy. Journal of proteomics, 2021. 236: p. 104124.

27. Takenaka, A., et al., An enzyme (beta-D-N-acety/glucosaminidase) released from the decidua to maternal plasma prior to the onset of labor. Acta Obstet Gynecol Scand, 1991. 70(6): p. 469-73.

28. Davison, J.M., et al., Serial evaluation of vasopressin release and thirst in human pregnancy. Role of human chorionic gonadotrophin in the osmoregulatory changes of gestation. J Clin Invest, 1988. 81(3): p. 798-806.

29. Quinn, P. and M. Petric, Anticomplementary activity in serum of women with a history of recurrent pregnancy loss. American journal of obstetrics and gynecology, 1988. 158(2): p. 368-72.

30. Singh, J., A. Ahmed, and G. Girardi, Role of complement component C1q in the onset of preeclampsia in mice. Hypertension (Dallas, Tex. : 1979), 2011. 58(4): p. 716-24.

31. Madhukaran, S.P., et al., Role of collectins and complement protein C1q in pregnancy and parturition. Immunobiology, 2016. 221(11): p. 1273-88.

32. Bulla, R., et al., Decidual endothelial cells express surface-bound C1q as a molecular bridge between endovascular trophoblast and decidual endothelium. Molecular immunology, 2008. 45(9): p. 262940.

33. Fliser, D. and E. Ritz, Serum cystatin C concentration as a marker of renal dysfunction in the elderly. Am J Kidney Dis, 2001. 37(1): p. 79-83.

34. Thilaganathan, B., et al., Early-pregnancy multiple serum markers and second-trimester uterine artery Doppler in predicting preeclampsia. Obstet Gynecol, 2010. 115(6): p. 1233-1238.

35. Holmes, V.A., Changes in haemostasis during normal pregnancy: does homocysteine play a role in maintaining homeostasis? Proc Nutr Soc, 2003. 62(2): p. 479-93.

\section{Figures}



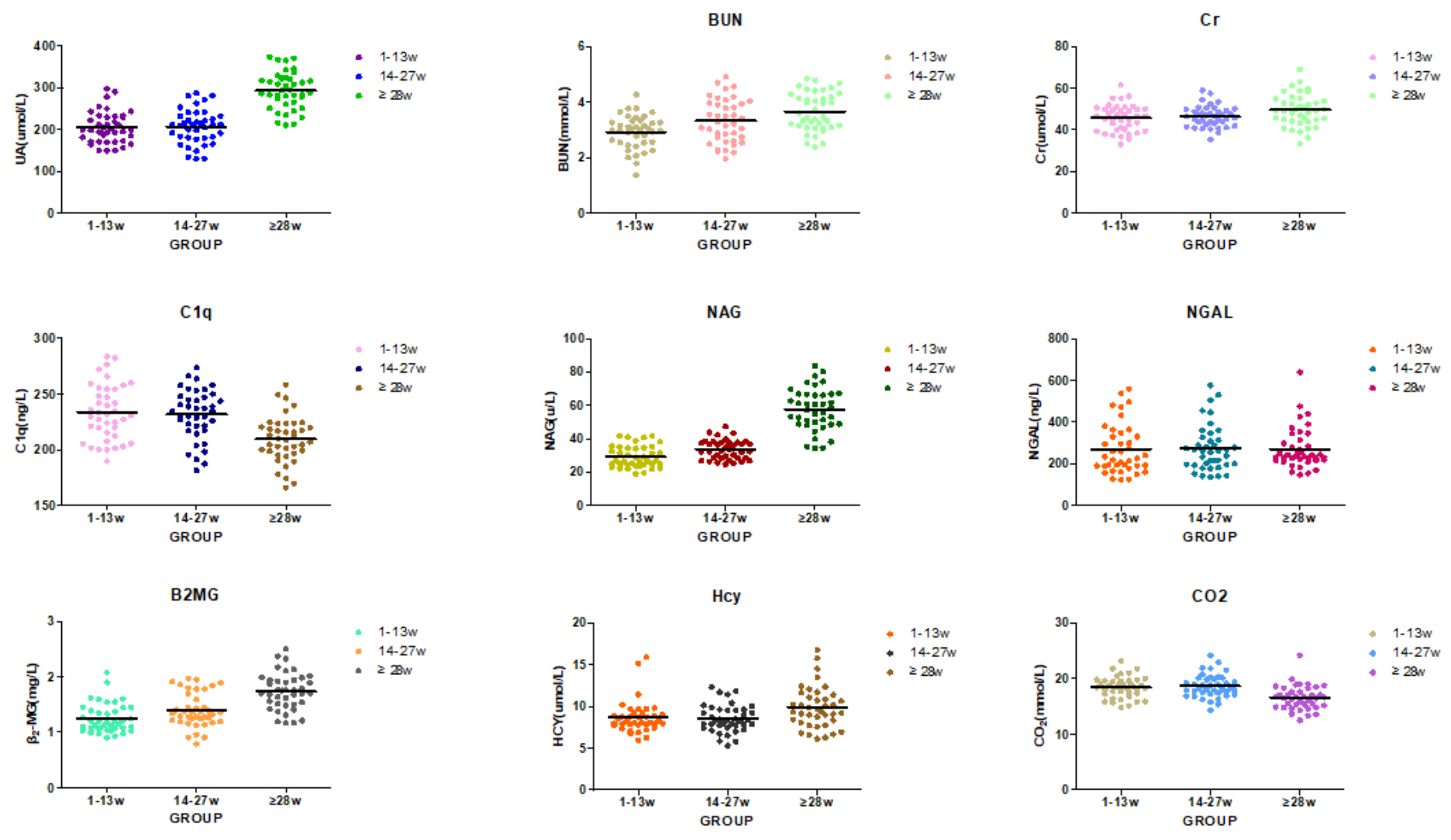

\section{Figure 1}

Significant changes of UA, NAG and NGAL, BUN, Cr, $\beta 2-M G, C O 2$, Hcy and C1q levels from the first trimester to the third trimester.(The black lines represent means) 